EPJ Web of Conferences 52, 10004 (2013)

DOI: $10.1051 /$ epjconf/20135210004

(C) Owned by the authors, published by EDP Sciences, 2013

\title{
Generation of Cosmic rays in Historical Supernova Remnants
}

\author{
V.G. Sinitsyna ${ }^{1, a}$ and V.Y. Sinitsyna ${ }^{1}$ \\ ${ }^{1}$ P. N. Lebedev Physical Institute, Russian Academy of Science
}

\begin{abstract}
We present the results of observations of two types of Galactic supernova remnants with the SHALON mirror Cherenkov telescope of Tien-Shan high-mountain Observatory: the shell-type supernova remnants Tycho, Cas A and IC 443; plerions Crab Nebula, 3c58(SN1181) and Geminga (probably plerion). The experimental data have confirmed the prediction of the theory about the hadronic generation mechanism of very high energy $(800 \mathrm{GeV}-100 \mathrm{TeV})$ gamma-rays in Tycho's supernova remnant. The data obtained suggest that the very high energy gamma-ray emission in the objects being discussed is different in origin.
\end{abstract}

\section{Introduction}

The hypothesis that Supernova Remnants (SNRs) are unique candidates for cosmic-ray sources $[1,2]$ has been prevalent from the very outset of cosmic-ray physics. Recent observations of several SNRs in X-rays and TeV gamma-rays will help in solving the problem of the origin of cosmic rays and are key to understanding the mechanism of particle acceleration at a propagating shock wave. A number of nearby Northern Hemisphere SNRs (see table 1) of different types has been observed in $\mathrm{TeV}$ energies with SHALON Cherenkov telescope at high altitude Observatory; some of them have been studied in details and the results of observations are presented in this paper.

\section{Crab Nebula (SN 1054)}

The Crab Nebula is the most famous Supernova Remnant. It plays an important role in the modern astrophysics. No other space object has such impact on the progress and development of the modern experimental and theoretical astrophysics methods.

Pulsar located at the center of the Crab Nebula is the first who's optical, X-ray and gamma-emission has been detected. Since the first detection with ground based telescope the Crab has been observed by the number of independent groups using different methods of registration of gamma-initiated showers [3-15]. Perhaps the most important fact is that this source with a stable flux can be used to calibrate Cherenkov telescopes in both Northern and Southern Hemispheres.

However, quite recently, the AGILE [16] and Fermi LAT [17] satellite experiments have reported on a flare exceeding the nominal flux from the Crab in the energy range

\footnotetext{
ae-mail: sinits@sci.lebedev.ru
}

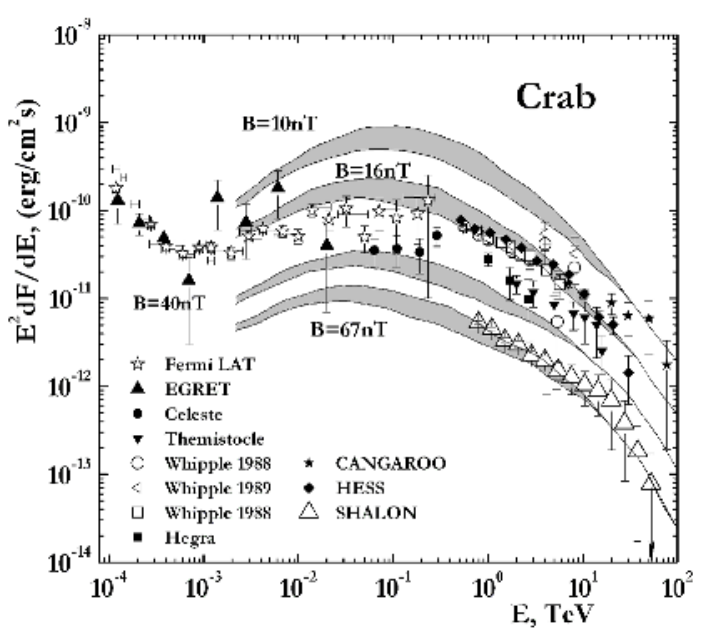

Figure 1. The Crab spectral energy distribution by SHALON [3-5] in comparison with other experiments [4-15].

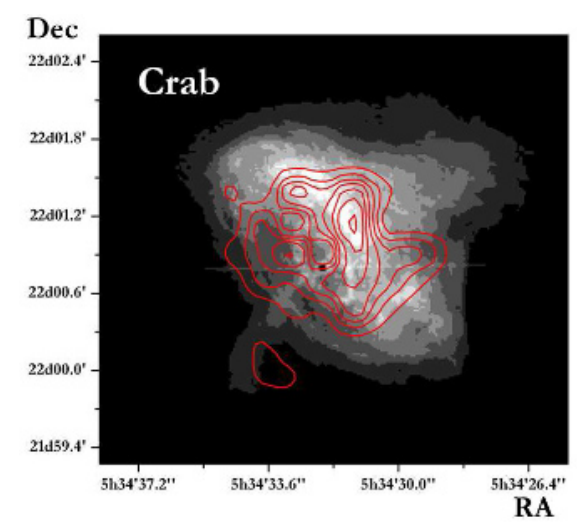

Figure 2. Chandra X-ray image of Crab [21]. The central part 200 " $\times 200$ " of Crab PWN in the energy range $0.2-4 \mathrm{keV}$. The red lines show the $\mathrm{TeV}$ - structure by SHALON observations.

from $100 \mathrm{MeV}$ to $2-5 \mathrm{GeV}$ by a factor of 4 , which was assumed to be absolutely stable and, consequently, was used 
Table 1. SNRs observed by SHALON at energies $>800 \mathrm{GeV}$.

\begin{tabular}{cccc}
\hline Sources & Type & Observable flux, $\left(\mathrm{cm}^{-2} \mathrm{~s}^{-1}\right)$ & Distance, $(\mathrm{kpc})$ \\
\hline Crab Nebula & Plerion & $(2.12 \pm 0.11) \times 10^{-12}$ & 2 \\
Geminga & Radioweak pulsar/Plerion & $(0.48 \pm 0.07) \times 10^{-12}$ & 0.25 \\
3c58(SN1181) & Plerion & $(0.84 \pm 0.33) \times 10^{-12}$ & $2.6-3.2$ \\
Tycho's SNR & Shell-type SNR & $(0.52 \pm 0.04) \times 10^{-12}$ & $3.1-3.3$ \\
Cas A & Shell type SNR & $(0.68 \pm 0.13) \times 10^{-12}$ & 3.4 \\
IC 443 & Shell-type SNR & $(1.69 \pm 0.58) \times 10^{-12}$ & 1.5 \\
\hline
\end{tabular}

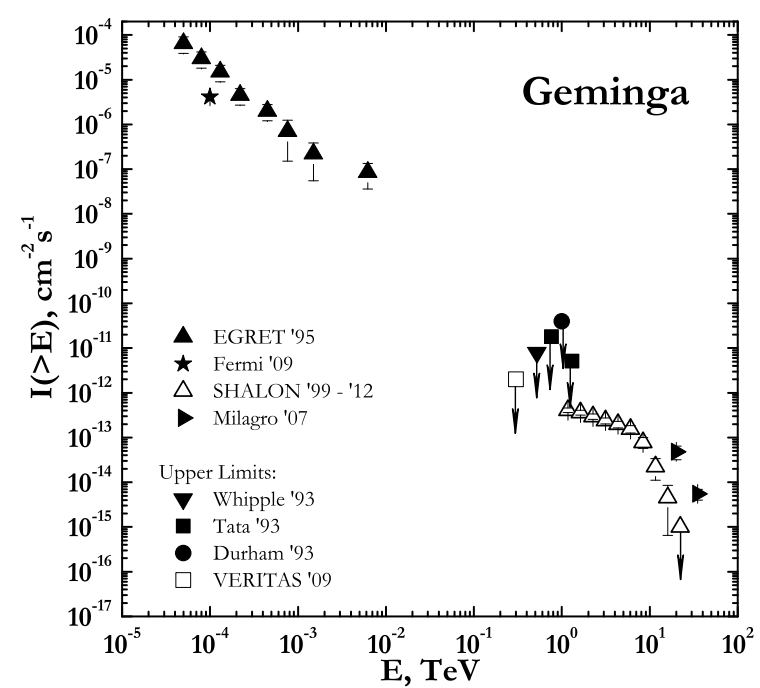

Figure 3. The Geminga integral $\gamma$ - quantum spectrum by SHALON in comparison with other experiments.

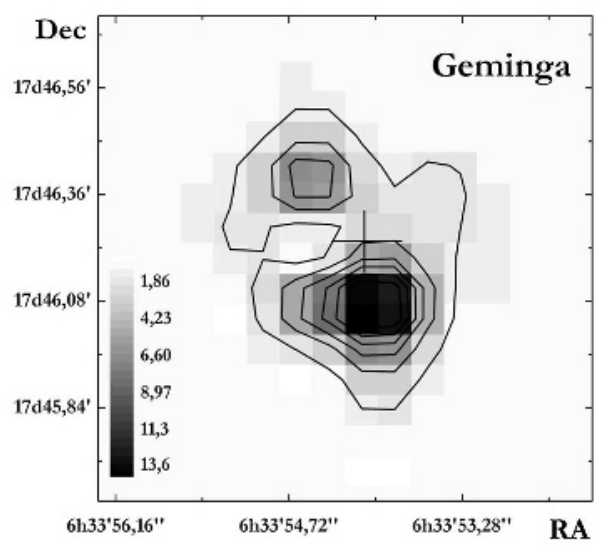

Figure 4. The image of $\gamma$-ray emission from Geminga by SHALON.

as a "standard candle". No flux increase was detected in the observations of the MAGIC [18] and VERITAS [19] ground-based telescopes in the same period. The spectrum of $\gamma$-rays from the Crab has been measured in the energy range $800 \mathrm{GeV}$ to $30 \mathrm{TeV}$ at the SHALON Cherenkov telescope [3-5] with a statistical significance [20] of 36.1 $\sigma$. The integral energy spectrum is well described by the single power law $I\left(>E_{\gamma}\right) \propto E_{\gamma}^{-1.40 \pm 0.07}$ (Fig. 1).

To made a description of the intensity and spectral shape in the $\mathrm{TeV}$ region, the model of Inverse Compton (IC) scattering of the ambient photons in the nebula in the Ref. [9] is used. Additionally, we need the assuming about magnetic field strength in the region of $\mathrm{TeV}$ emission (Fig. 2, right). The average magnetic field in the region of $\mathrm{TeV} \gamma$-ray emission is extracted from the comparison of $800 \mathrm{GeV}-30 \mathrm{TeV}$ (SHALON data) and X-ray (Chandra data [21]) emission regions (Fig. 2).

Figure 2 presents a Chandra X-ray image of the central part 200 " $\times 200$ " of Crab Nebula in the energy range $0.2-$ $4 \mathrm{keV}$ [21]. In this energy band most of the PWN X-rays come from a torus surrounding the pulsar. The contour lines show the TeV - structure by SHALON observations. The most part of $\mathrm{TeV}$ energy $\gamma$-quanta come from the region of bright torus (see Fig. 2). Magnetic fields and lifetimes of representative regions in Chandra image of Crab have been derived [21] and ranges ranges from $62 \mathrm{nT}$ up to $153 \mathrm{nT}$ with the average value $67 \pm 7 \mathrm{nT}$. The $\gamma$-ray emission regions observed by SHALON in the Crab correlate well with the emission regions of synchrotron photons in the energy range $0.4-2.1 \mathrm{keV}$.

Finally, the TeV $\gamma$-ray spectrum of Crab by SHALON is generated via IC scattering of soft, mainly optical, photons which are produced by relativistic electrons and positrons, in the nebula region around 1.5 '(Fig. 2) from the pulsar with specific average magnetic field of about $67 \pm 7 \mathrm{nT}$.

\section{Geminga( $\sim 3.4 \times 10^{5}$ yr $)$}

A neutron star in the constellation Gemini is the second brightest source of high-energy $\gamma$-rays in the sky, discovered in 1972, by the SAS-2 satellite. For nearly 20 years, the nature of Geminga was unknown, since it didn't seem to show up at any other wavelengths. In 1991, an regular periodicity of 0.237 second was detected by the ROSAT satellite in soft X-ray emission, indicating that Geminga is almost certainly a pulsar. Geminga is the closest known pulsar to Earth. It is one of the brightest source of $\mathrm{MeV}$ $\mathrm{GeV} \gamma$-ray, but the only known pulsar that is radio-quiet. Also this object it suggested to be a Geminga supernova remnant with expected age of $\sim 3.4 \times 10^{5} \mathrm{yr}[22,23]$. The 


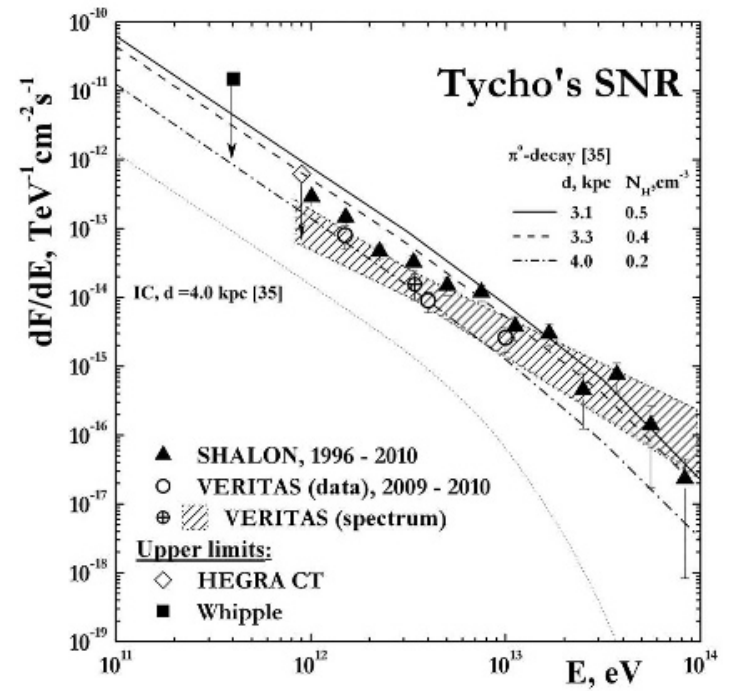

Figure 5. The differential spectrum of Tycho's SNR.

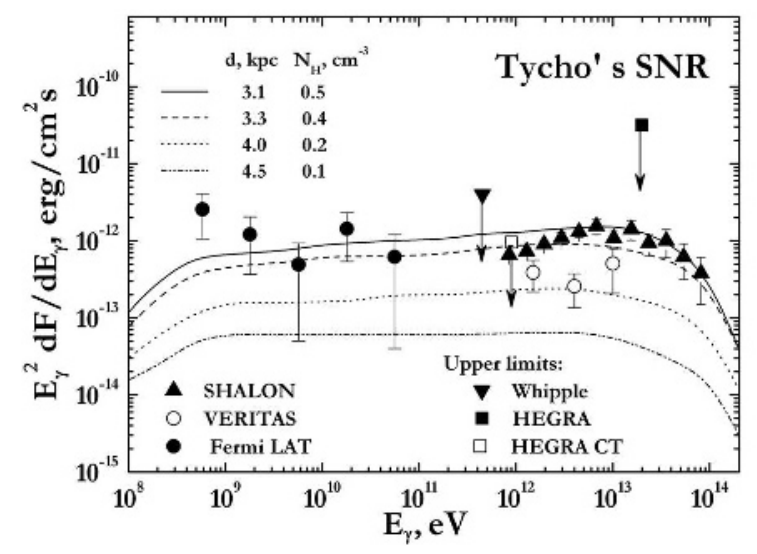

Figure 6. Spectral energy distribution of the $\gamma$-ray emission from Tycho's SNR.

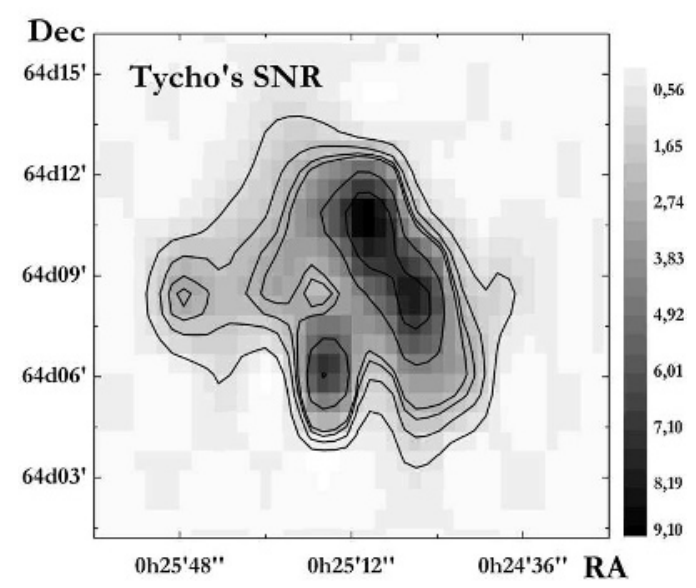

Figure 7. The Tycho's SNR image in TeV $\gamma$-rays by SHALON.

extended VHE emission from Geminga (in Fermi observations) could arise from the Pulsar Wind Nebula associated with the Geminga SNR remnant.
Geminga has been the object for study at $\mathrm{TeV}$ energies with upper limits being reported by three experiments Whipple'93 [24], Tata'93 [25], Durham'93 [26] and very recently by VERITAS'09 [27]. Also Geminga has observed with Milagro'07 [28, 29] at energies of $20 \mathrm{TeV}$ and $35 \mathrm{TeV}$ and Fermi LAT at energies $30 \mathrm{MeV}$ - 200GeV [30].

The observations of Geminga by SHALON in 1999 year had yielded the detection of $\gamma$-ray emission from this object [3-5] and it have been intensively studied since then. Figures 3 and 4 show the SHALON results for this $\gamma$-source [3-5]. As is seen from this figure the value Geminga flux obtained by SHALON is lower than the upper limits published before. Its integral $\gamma$-ray flux is found to be $(0.48 \pm 0.07) \times 10^{-12} \mathrm{~cm}^{-2} \mathrm{~s}^{-1}$ at energies of $>0.8$ $\mathrm{TeV}$ [3] with a significance of $7.6 \sigma$ [20]. An image of $\gamma$-ray emission from Geminga by SHALON telescope is shown in Fig. 4. Within the range $0.8-6 \mathrm{TeV}$, the integral energy spectrum is well described by the single power law $I\left(>E_{\gamma}\right) \propto E_{\gamma}^{-0.59 \pm 0.10}$ (Fig. 3). The energy spectrum of supernova remnant Geminga $F\left(E_{O}>0.8 T e V\right) \propto E^{k}$ is harder than Crab spectrum.

\section{Tycho's SNR (SN 1572)}

Tycho supernova remnant has been observed by by SHALON atmospheric Cherenkov telescope of Tien-Shan high-mountain observatory since 1996 (Figs. 5, 6, 7) [35]. This object has long been considered as a candidate to cosmic ray hadrons source in Northern Hemisphere.

Tycho's SNR has been detected by SHALON at $\mathrm{TeV}$ energies [3-5] (in observations of 1996 - 2010 years) with a statistical significance [20] of $17 \sigma$. The integral $\gamma$-ray flux above $0.8 \mathrm{TeV}$ was estimated as $(0.52 \pm 0.04) \times 10^{-12} \mathrm{~cm}^{-2} \mathrm{~s}^{-1}$. The energy spectrum of $\gamma$-rays in the observed energy region from $0.8 \mathrm{TeV}$ is well described by the power law with exponential cutoff, $I\left(>E_{\gamma} / 1 \mathrm{TeV}\right)=(0.42 \pm 0.04) \times 10^{-12} \times$ $\left(E_{\gamma} / 1 \mathrm{TeV}\right)^{-0.93 \pm 0.09} \exp \left(-E_{\gamma} / 35 \mathrm{TeV}\right) \mathrm{cm}^{-2} \mathrm{~s}^{-1}$ (Fig. 5). An image of $\gamma$-ray emission from Tycho's SNR by SHALON telescope is shown in Fig. 7. Recently, Tycho's SNR was also confirmed with VERITAS [31] in observations of 2008 - 2010 years. The high energy $\gamma$-ray emission from Tycho'SNR was detected with Fermi LAT [32] in the range $400 \mathrm{MeV}-100 \mathrm{GeV}$ (Figs. 6).

A nonlinear kinetic model of cosmic ray acceleration in supernova remnants is used in $[33,34]$ to describe the properties of Tycho's SNR. The expected flux of $\gamma$-quanta from $\pi^{\circ}$-decay, $F_{\gamma} \propto E_{\gamma}^{-1}$, extends up to $\sim 30 \mathrm{TeV}$, while the flux of $\gamma$-rays originated from the IC scattering has a sharp cut-off above the few $\mathrm{TeV}$, so the detection of $\gamma$-rays with energies up to $80 \mathrm{TeV}$ by SHALON (Figs. 5, 6) is an evidence of their hadronic origin [33-35]. Figure 6 presents spectral energy distribution of the $\gamma$-ray emission from Tycho's SNR, as a function of $\gamma$-ray energy $\epsilon_{\gamma}$, for a mechanical SN explosion energy of $E_{S N}=1.2 \times 10^{51} \mathrm{erg}$ and four different distances $d$ and corresponding values of the interstellar medium number densities $N_{H}$. All cases have dominant hadronic $\gamma$-ray flux [35]. 


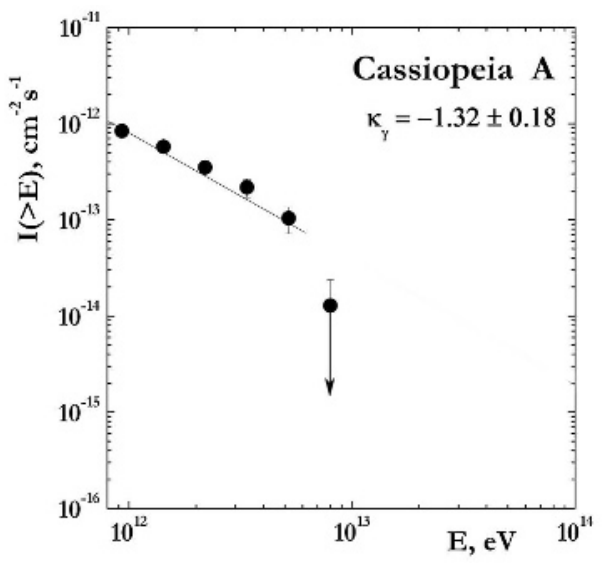

Figure 8. Cas A $\gamma$-ray integral spectrum by SHALON experiment.

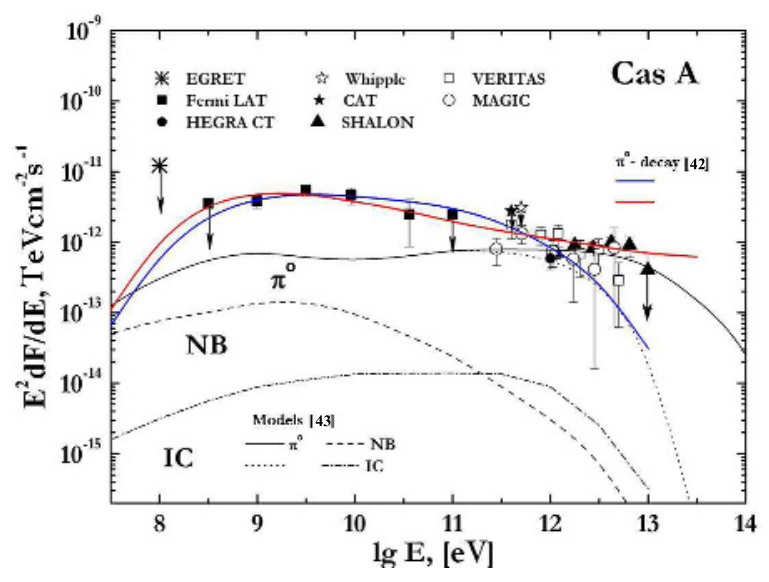

Figure 9. Spectral energy distribution of the $\gamma$-ray emission from Cas A.

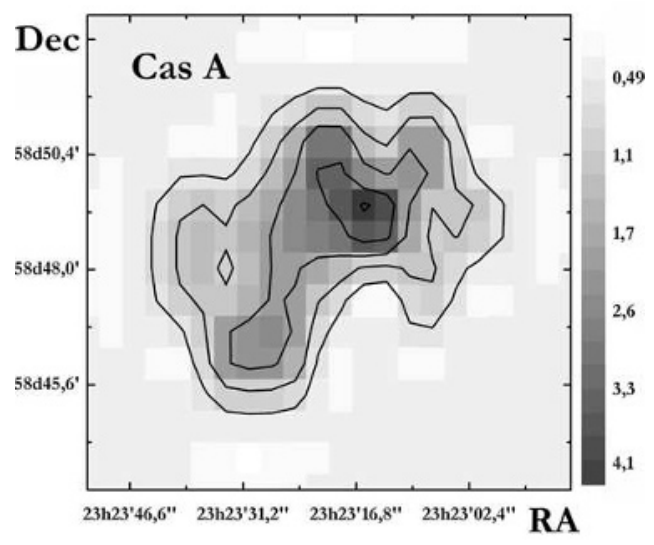

Figure 10. The Cas A image in $\mathrm{TeV} \gamma$-rays by SHALON.

The additional information about parameters of Tycho's SNR can be predicted in frame of nonlinear kinetic model if the TeV $\gamma$ - quantum spectrum of SHALON telescope is taken into account: a source distance $3.1-3.3 \mathrm{kpc}$ and an ambient density $N_{H}$ is about $0.5-0.4 \mathrm{~cm}^{-3}$ and the expected $\pi^{\circ}$-decay $\gamma$-ray energy spectrum extends up to about $100 \mathrm{TeV}$ [33-35].

\section{Cassiopeia A (SN 1680)}

Cassiopeia A (Cas A) is the youngest of historical supernova remnant in our Galaxy. The supernova explosion that gave rise to Cas A occurred around 1680. Its overall brightness across the electromagnetic spectrum makes it a unique object for studying high-energy phenomena in SNRs [5, 36-42]. Cas A was detected in TeV $\gamma$ rays, first by HEGRA'01 [36] and later confirmed by MAGIC'07 [37] and VERITAS' 10 [38]. The high energy $\gamma$-ray emission from Cas A was detected with Fermi LAT [42] in the range $500 \mathrm{MeV}-50 \mathrm{GeV}$.

Cas A was observed with SHALON telescope during the 27 hours of autumn 2010 [5]. All observations were made with the standard procedure of SHALON experiment during moonless nights with zenith angles from 13 to 35 degree. The $\gamma$-ray source associated with the SNR Cassiopeia A was detected above $800 \mathrm{GeV}$ (Figs. 8, 9, 10) with a statistical significance of $7.1 \sigma$ [20] with a $\gamma$-quantum flux of $I_{\text {CasA }}(>0.8 T e V)=(0.68 \pm 0.13) \times 10^{-12} \mathrm{~cm}^{-2} \mathrm{~s}^{-1}$. The $\gamma$-ray integral spectrum is presented in Fig. 8 , it is compatible with a power law with an index $k_{\gamma}=1.32 \pm$ 0.18 in energy range of $0.8-8 \mathrm{TeV}$ (Fig. 8).

The favored scenarios in which the $\gamma$-rays of $500 \mathrm{MeV}$ - $10 \mathrm{TeV}$ energies are emitted in the shell of the SNR like Cas A are considered in [42, 43]. The $\gamma$-ray emission could be produced by electrons accelerated at the forward shock through relativistic bremsstrahlung (NB) or IC [42]. Alternatively, the $\mathrm{GeV} \gamma$-ray emission could be produced by accelerated CR hadrons through interaction with the background gas and then $\pi^{\circ}$-decay.

Figure 9 presents spectral energy distribution of the $\gamma$-ray emission from Cas A by SHALON in comparison with other experiment data Fermi LAT [42], HEGRA [36], MAGIC [37], VERITAS [38], EGRET [39], CAT [40], Whipple [41] and with theoretical predictions [42], [43]. Solid lines show the very high energy $\gamma$-ray spectra of hadronic origin. Dash-and-dot line presents the $\gamma$ ray spectrum of the IC emission [42]. The leptonic model with $\mathrm{B}=0.12 \mathrm{mG}$ and $\mathrm{B}=0.3 \mathrm{mG}$ also proposed in [42]. It was shown that leptonic model with $\mathrm{B}=0.3 \mathrm{mG}$ predicts a 5 - 8 times lower $\gamma$-ray flux than the observed; the model with $\mathrm{B}=0.12 \mathrm{mG}$, which can broadly explain the observed $\mathrm{GeV}$ flux predicts the $\mathrm{TeV}$ spectrum with cut-off energy about $10 \mathrm{TeV}$.

The detection of $\gamma$-ray emission at $5-10 \mathrm{TeV}$ and the hard spectrum below $1 \mathrm{TeV}$ would favor the $\pi^{\circ}$-decay origin of the $\gamma$-rays in Cas A SNR.

\section{Conclusion}

The investigation of VHE gamma-ray sources by any methods, including mirror Cherenkov telescopes, touches on the problem of the cosmic ray origin and, accordingly, the role of the Galaxy in their generation. The SHALON observations at the Tien Shan high-altitude observatory 
yielded data on four SNRs: the Crab Nebula, Geminga, Cassiopeia A, and Tycho at energies above $0.8 \mathrm{TeV}$. The data obtained suggest that the very high energy gammaray emission in the objects being discussed is different in origin.

\section{References}

[1] E. G. Berezhko and G. F. Krymskii, Usp. Fiz. Nauk 154(1), 49 (1988)

[2] S. P. Reynolds, Ann. Rev. Astron. Astrophys. 46, 89 (2008)

[3] V. G. Sinitsyna, AIP (Conf. Proc.) 515, 205, 293 (2000)

[4] V. G. Sinitsyna et al., Nucl. Phys. B (Proc.Suppl.) 196, 437 (2009); ibid. 175-176, 455 (2008); ibid. 151, 112 (2006); ibid. 122, 247, 409 (2003); ibid. 97, 215, 219 (2001); ibid. 75A, 352 (1999)

[5] V. G. Sinitsyna and V. Yu. Sinitsyna, Astron. Lett. 37(9), 621 (2011).

[6] T. C. Weekes, AIP Conf. Proc. 515, 3 (1999).

[7] M. Catanese \& T.C.Weekes, Prep. Ser., No4811 (1999).

[8] J. Prahl and C.Prosch (for the HEGRA Collaboration), in Proc. 25th ICRC, Durban, 3, 217 (1997).

[9] A. M. Hillas, et al., ApJ 503, 744 (1998).

[10] T. Tanimori, et al., ApJ 492, 133 (1998).

[11] F. Piron, et al., in Proc. 28th ICRC, Tsukuba 2607 (2003).

[12] F. Aharonian, et al. ApJ 539, 317 (2000).

[13] C. Masterson, et al., in Proc. 29th ICRC, Puna 4, 143 (2005).

[14] A. A. Abdo, et al., ApJ 708, 1254 (2010).

[15] R. M. Wagner, et al., in Proc. 29th ICRC, Puna 4, 163 (2005).

[16] M. Tavani et al., Science 331, 736 (2010)

[17] A, A. Abdo et al., Science 331(6018), 739 (2010)

[18] M. Mariotti Astron. Telegramm No. 2967 (2010)

[19] R. Ong Astron. Telegramm No. 2968 (2010)
[20] T.-P. Li and Y.-Q. Ma, Astrophys. J. 272, 317 (1983)

[21] F. D. Seward, W. H. Tucker and R, A. Fesen, Astrophys. J. 652, 1277 (2006)

[22] M. Salvati, B. Sacco, Astron.\&Astrophys. 485, 527 (2008)

[23] M. Salvati, Astron.\&Astrophys. 513, A28 (2010)

[24] C. W. Akerlof et al., in Proc. 23rd ICRC, Calgary 1, 305 (1993)

[25] P. R. Vishwanath et al., Astron.\&Astrophys. 267, L5 (1993)

[26] C. C. G. Bowden et al., J. Phys. G: Nucl. Part. Phys. 19, L29 (1993)

[27] G. Finnegan, in Proc. of the 31st ICRC, Lodz, (2009)

[28] A. A. Abdo et al., Astrophys.J. 700, L127-L131, (2009);

[29] A. A. Abdo et al., Astrophys.J. 664, L91-L94 (2007)

[30] O. Celik, in Proc. of the 31st ICRC, Lodz, (2009)

[31] V. A. Acciari et al., Astroph. J. Lett. 730, L20 (2011).

[32] F. Giordano et al., Astroph. J. Lett. 744(1), L2 (2011).

[33] H. J. Völk et al., in Proc. 27th ICRC, Hamburg 2, 2469 (2001)

[34] E. G. Berezhko et al., Astrophys. Space Sci. 309, 385 (2007)

[35] H. J. Völk, E. G. Berezhko, L, T. Ksenofontov, Astron.\&Astrophys. 483(2), 529 (2008)

[36] F. Aharonian et al., Astron.\&Astrophys. 370, 112 (2001)

[37] J. Albert et al., Astron.\&Astrophys. 474, 937 (2007)

[38] V. A. Acciari et al., Astrophys. J. 714, 163 (2010)

[39] J. A. Esposito et al., Astrophys. J. 461, 820 (1996).

[40] P. Goret, et al., Proc. of 26th ICRC, (Ed. D. Kieda, M. Salamon, B. Dingus) 3, 496 (1999).

[41] R. W. Lessard, et al., Proc. of 26th ICRC, (Ed. D. Kieda, M. Salamon, B. Dingus) 3, 488 (1999).

[42] A. A. Abdo et al., Astrophys. J. 710, L92 (2010)

[43] E. G. Berezhko, G. Pühlhofer, H. J. Völk, Astron.\&Astrophys 400, 971 (2003) 